A viscoelastic damage model for polycrystalline ice, inspired by Weibull-distributed fiber bundle models. Part I: Constitutive models

Journal Article

Author(s):

Keller, Arne; Hutter, Kolumban (1)

Publication date:

2014-11

Permanent link:

https://doi.org/10.3929/ethz-b-000081888

Rights / license:

In Copyright - Non-Commercial Use Permitted

Originally published in:

Continuum Mechanics and Thermodynamics 26(6), https://doi.org/10.1007/s00161-014-0348-7 


\title{
A viscoelastic damage model for polycrystalline ice, inspired by Weibull-distributed fiber bundle models. Part I: Constitutive models
}

Received: 16 October 2013 / Accepted: 3 March 2014 / Published online: 1 April 2014

(C) Springer-Verlag Berlin Heidelberg 2014

\begin{abstract}
We consider a constitutive model for polycrystalline ice, which contains delayed-elastic and viscous deformations, and a damage variable. The damage variable is coupled to the delayed-elastic deformation by a fiber bundle ansatz. We construct an isotropic theory, which can be calibrated with experimental data. Furthermore, we generalize the theory to a damage model in terms of rank-four tensors. This general model allows the evolution of anisotropic damage.
\end{abstract}

Keywords Polycrystalline ice · Viscoelastic damage · Delayed-elastic response • Rank-4 . damage variable . Weibull-distributed fiber bundle model

\section{Introduction}

The deterioration and failure of polycrystalline ice is an important subject in glaciological research. It is of high interest in geophysical contexts, such as glacier and ice shelf calving or sea ice mechanics, and also for engineering applications.

The most common approach to deterioration and failure of ice is continuum damage mechanics. In such formulations, two different approaches are taken to quantify damage. The first approach considers defects on the microscale, describes these defects in terms of their size and/or orientation and employs statistical averaging to describe the effect of these defects on the larger scale. This approach is favored in the books [10] and [14]. In the second approach, damage is described by a phenomenological variable, scalar valued for isotropic damage and tensor valued for anisotropic damage $[1,7,10,12,19]$. With a view focussing on damage evolution in creeping deformation of polycrystalline ice, continuum damage mechanics has been applied to describe progressive accumulation of microcracks in [7,13,15,20-22]. In these works, one adds to the physical fields of continuum mechanics an internal variable, which by use of suitably constructed balance and constitutive equations can be interpreted either as a damage variable $D$ (which is equal to zero for the virgin material and unity for the completely damaged material) or as damage effect variable $Z$ (which equals unity for the virgin material and, with increasing damage, monotonically grows without bound). Both variables must be regarded as tensors of certain rank (depending on the level of anisotropy under consideration). In the isotropic case, $D$ can be interpreted as the ratio of damaged to total cross-sectional area. Correspondingly, $Z=1 /(1-D)$

Communicated by Andreas Öchsner.

A. Keller $(\varangle) \cdot$ K. Hutter

Laboratory of Hydraulics, Hydrology and Glaciology (VAW), ETH Zürich, Wolfgang-Pauli-Str. 27, 8093 Zurich, Switzerland E-mail: keller@vaw.baug.ethz.ch

Tel.: +41446326611

K. Hutter

E-mail: hutter@vaw.baug.ethz.ch

Tel.: +41446324123 
is the ratio of total to un-damaged cross-sectional area (see, e.g., [15]). For higher anisotropy, the physical interpretation of the damage variables is not so obvious and becomes clear only with the choice of constitutive relations. Furthermore, for anisotropic damage, particular care has to be taken in order not to violate the second law of thermodynamics. It has been pointed out by Ref. [8] that anisotropic damage should be modeled by a rank-4 damage effect tensor of a certain structure in order to assure thermodynamic consistency; a requirement which we will fulfill in the model presented in this work.

Recently, a new viscoelastic damage model for ice has been published [3]. This model, however, uses an additive coupling of the strain tensors. This approach is applicable only if all deformations-including viscous deformations - can be considered as small, which is certainly not the case for glaciological applications. The damage model presented in the following does not suffer from this conceptual shortcoming.

Independent of the choice of the damage variable, the delicate part of designing a reasonable damage theory is to establish a suitable constitutive framework. Usually, this will consist of two steps: At first, one has to properly define how the damage degree of freedom is embedded in the constitutive behavior of the undamaged material; afterward, the balance equation for damage has to be closed by constructing a damage evolution function. As a constitutive quantity, the latter has to be constructed in a way both reflecting experimental observations and respecting certain general physical principles (e.g., the entropy principle).

In the following, we will consider a slightly different approach, which avoids the problem of stating a suitable damage evolution law. Instead of treating damage as one or several dynamical degree(s) of freedom, we assume it to be a function of the delayed-elastic deformation (i.e., the component of deformation which is recoverable but not instantaneous). This approach to ice damage has first been proposed by Ref. [18] This author carried out deformation experiments on samples of artificial polycrystalline ice, while optically observing the crack density. He came to the conclusion that damage seems to be governed by delayed elasticity in polycrystalline ice.

In this approach, the crucial question is not how to establish a damage evolution law, but how to define a suitable damage function depending on delayed elasticity. For this, we will use a so-called fiber bundle model. The latter originates from the idea that the material consists of a big number of entities which can break at a certain (more or less random) threshold stress or deformation. Such procedures are very common in the context of statistical damage models (see e.g., [11]). We will combine in the following a mean-field fiber bundle approach with a nonlinear viscoelastic rheological model.

The advantage of our approach is - apart from implementing the postulate, that damage should be coupled to delayed elasticity-that it has (1) the simple interpretation of a fiber bundle model, (2) it allows a rather straightforward anisotropic generalization, (3) it is technically handy for the evaluation of its thermodynamic consistency. Furthermore, we pay much attention to the kinematics of a tensorial model and solve the problem that an additive coupling of the strain tensors (such as used by, e.g., [18] and [3]) is not feasible in the presence of large viscous deformations. The model presented in the following does not include healing of pre-existing damage. The latter is a very delicate subject, on which to our knowledge no experimental data are available for polycrystalline ice. Nevertheless, the fact that for our damage model, the thermodynamics are well known (see part II, [9]) has to be seen as basis for inclusion of a healing model in a thermodynamically reasonable way.

The structure of this article is as follows: At first, we will discuss the general idea of our approach to deterioration and failure of ice. We will illustrate the basic features of our models with a rather abstract onedimensional example. As, nevertheless, a calibration is necessary, we will give a straightforward generalization of the one-dimensional example to an isotropic theory, which can handily be calibrated with experimental data.

As a further generalization, we will include the theory in a constitutive framework for anisotropic ice damage. This requires a considerable amount of extra effort, mostly due to the non-obvious coupling of delayed-elastic and viscous strains.

Finally, let us alert the reader that our approach from simple situations, step by step, to the general threedimensional constitutive model is in many points motivation, and not derivation or deduction. This should be kept in mind, even though quite advanced mathematical steps might be involved in the actual pursuit of the generalization.

\section{Isotropic damage model}

In this section, an isotropic damage model for polycrystalline ice will be discussed. We will at first discuss the basic concepts (strain splitting, rheological model, fiber bundle principle) on a one-dimensional example. 


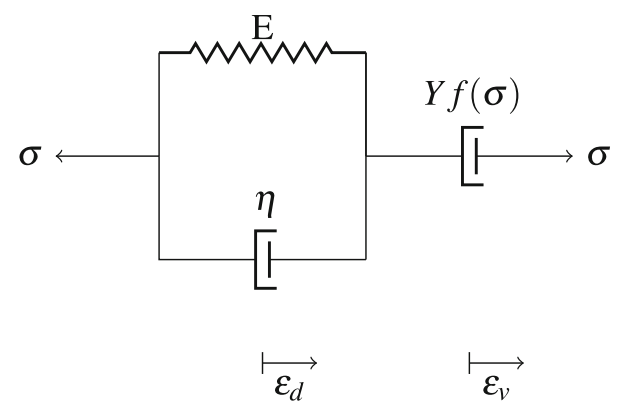

Fig. 1 Spring-dashpot diagram of the used rheological model. The left part constitutes the delayed-elastic deformation component $\varepsilon_{d}$, whereas the right part represents the viscous component $\varepsilon_{v}$

Even though helpful for this purpose, a one-dimensional model is of no practical use and cannot be calibrated either. Therefore, we generalize it to an isotropic model by applying the one-dimensional model to isotropic shear and bulk deformations.

\subsection{General concept of the fiber bundle damage model}

In the following, we will symbolically speak about "the strain" as a one-dimensional quantity. This "strain" $\varepsilon$ can be any dimensionless one-dimensional deformation measure and merely serves to explain the basic ideas of our material model. We will in a later section, apply the theory to rigorously defined deformation measures.

We assume that the total strain $\varepsilon$ consists of elastic, delayed-elastic and viscous deformations,

$$
\varepsilon=\varepsilon_{e}+\varepsilon_{d}+\varepsilon_{v}
$$

The elastic deformation $\varepsilon_{e}$ is instantaneous, and we assume it not to be coupled to the other deformations. We will ignore this component, as it is likely small compared with $\varepsilon_{d}$ and $\varepsilon_{v}$, and does not have any influence on creep deformation. It can, however, without further difficulties be introduced by simply assuming linear elastic behavior,

$$
\varepsilon_{e}=C \sigma,
$$

where $\sigma$ is the stress and $C$ a compliance constant.

For the delayed-elastic strain $\varepsilon_{d}$ of every single fiber, we use a Kelvin-Voigt unit (as depicted in the left part of Fig. 1), which contains a damage variable that adds a non-linearity as follows:

$$
\dot{\varepsilon}_{d}=\frac{1}{\eta}\left(Z \sigma-E \varepsilon_{d}\right)
$$

where $\eta$ is a viscosity and $E$ Young's modulus. ${ }^{1} Z$ plays the role of stress reduction/enhancement due to damage and is analogous to the damage effect variable being introduced later on.

In order to allow the material to deteriorate, we consider it to consist of a large number of fibers, which may break with growing deformation. Each fiber has a certain deformation threshold at which it will break. The thresholds are assumed to be Weibull- distributed; i.e., the expectation value for the fraction of broken fibers at a deformation $\varepsilon$ reads

$$
P(\varepsilon)=1-\exp \left[-\left(\frac{\varepsilon}{\lambda}\right)^{\rho}\right]
$$

with parameters $\lambda$ and $\rho$. Equation (4) states that, as the material is deforming, more and more fibers reach their threshold deformation, and will subsequently be destroyed. Thus, the material is subject to damage. So far, we did not specify which component of the deformation is relevant to the distribution given by Eq. (4). Motivated by Ref. [18], we assume damage to be governed by the delayed-elastic deformation $\varepsilon_{d}$. Consequently, we will in the following replace $\varepsilon$ in Eq. (4) by $\varepsilon_{d}$. This is a central assumption of the damage model presented here.

\footnotetext{
1 This modulus and viscosity are the quantities which correspond to the abstract strain $\varepsilon$. Strictly defined moduli which correspond to real deformation components will be introduced in a later section.
} 


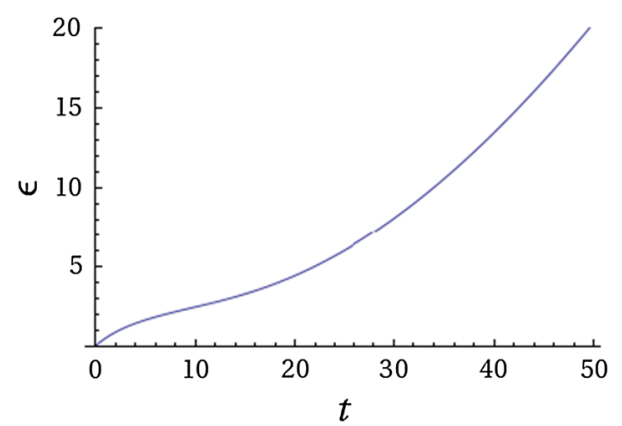

Fig. 2 Example plot for strain evolution $\varepsilon(t)=\varepsilon_{d}+\varepsilon_{v}$. Arbitrary units for $t$. Parameters (arbitrary units): $\sigma=2.6, \eta=5$, $E=1.4, \rho=2, \lambda=5, v=100$. Creep enhancement factor $Y_{1}$ with $\beta=5$

The damage effect $Z$ is the factor by which the stress in a single fiber is enhanced with respect to the undamaged case. For a fiber bundle model with democratic load sharing, it equals the fraction of total to intact fibers,

$$
Z=\frac{1}{1-P\left(\varepsilon_{d}\right)}=\frac{n_{\text {total }}}{n_{\text {total }}-n_{\text {broken }}} .
$$

For a mean-field model, i.e., in the limiting case of large numbers $n_{\text {total }}$, the number of broken fibers equals its expectation value, and we can write $Z$ as a function of the delayed-elastic deformation $\varepsilon_{d}$ as

$$
Z\left(\varepsilon_{d}\right)=\exp \left[\left(\frac{\varepsilon_{d}}{\lambda}\right)^{\rho}\right] \text {. }
$$

The nonlinear time evolution of the delayed-elastic strain is therefore given by

$$
\dot{\varepsilon}_{d}=\frac{1}{\eta}\left[\sigma \exp \left[\left(\frac{\varepsilon_{d}}{\lambda}\right)^{\rho}\right]-E \varepsilon_{d}\right] \text {. }
$$

The viscous deformation component $\varepsilon_{v}$ (represented by the dashpot to the right in Fig. 1) is assumed to behave like a (possibly nonlinear) viscous fluid, subject to damage. The deformation rate thus has to be written as a function of the stress $\sigma$ and the damage variable $Z$, for which we propose

$$
\dot{\varepsilon}_{v}=Y(Z) f(\sigma) \sigma .
$$

$f(\cdot)$ is a function that mimics the rheological behavior of the undamaged material, the most important choice being $f=v=$ const. for a Newtonian fluid with viscosity $v . Y(Z)$ is called the strain enhancement factor, which parameterizes how damage affects the rheology. This quantity allows a certain freedom for constitutive modeling; we propose to use either of

$$
\begin{aligned}
Y_{1} & =\frac{1}{1-\beta\left(1-Z^{-1}\right)}, \\
Y_{2} & =1+\beta\left(1-Z^{-1}\right), \\
Y_{3} & =\frac{1}{1-\beta \operatorname{artanh}\left(1-Z^{-1}\right)},
\end{aligned}
$$

with $\beta=$ const. In the limit $\beta\left(1-Z^{-1}\right) \ll 1$, these agree with one another. (Note: Eq. (10) is very similar to an ansatz proposed by [18]).

\subsection{Rheological effects, equilibrium states}

In the following, we will discuss the rheological properties of the proposed damage model. It will turn out that it produces primary, secondary and tertiary creep (an example is shown in Fig. 2). Apart from that, it has stable equilibrium states with constant damage, which can exist up to a certain threshold stress. These equilibrium states can be calculated analytically, together with the limits beyond which critical behavior occurs. As a remarkable fact it turns out that for a wide class of strain enhancement factors $Y$ in combination with Newtonian behavior for the undamaged material the subcritically damaged fluid has a regularized power-law rheology. 


\subsubsection{Equilibrium states}

If we set $\dot{\varepsilon}_{d}=0$ in Eq. (7), we obtain an equation for the equilibrium value of $\varepsilon_{d}$,

$$
\frac{\varepsilon_{d}^{e q}}{\lambda}-\alpha \exp \left[\left(\frac{\varepsilon_{d}^{e q}}{\lambda}\right)^{\rho}\right]=0,
$$

where $\alpha=\frac{\sigma}{E \lambda}$ is the dimensionless stress. This equation can be written as

$$
-\rho\left(\frac{\varepsilon_{d}^{e q}}{\lambda}\right)^{\rho} \exp \left[-\rho\left(\frac{\varepsilon_{d}^{e q}}{\lambda}\right)^{\rho}\right]=-\rho \alpha^{\rho},
$$

which has the solution

$$
\varepsilon_{d}^{e q}=\frac{\lambda}{\rho^{\frac{1}{\rho}}}\left[-W\left(-\rho \alpha^{\rho}\right)\right]^{\frac{1}{\rho}},
$$

where $W$ stands for the Lambert $W$-function ${ }^{2}$. The corresponding damage effect reads

$$
Z_{e q}=\frac{1}{\alpha \rho^{\frac{1}{\rho}}}\left[-W\left(-\rho \alpha^{\rho}\right)\right]^{\frac{1}{\rho}} .
$$

The limit of subcritical behavior is given by the point up to which the Lambert function in Eq. (14) has real values. This is the case if its argument is $\geq-\frac{1}{\mathrm{e}}$ (where $\mathrm{e}$ is the base of the natural logarithm); in terms of the stress $\sigma$ this yields

$$
\sigma \leq \sigma_{c}=E \lambda(\mathrm{e} \rho)^{-\frac{1}{\rho}} .
$$

Furthermore, we can study the behavior of the strain enhancement factor $Y$ in an equilibrium state. Making use of the expansion [2]

$$
W(x)=\sum_{n=1}^{\infty} \frac{(-n)^{n-1}}{n !} x^{n},
$$

which has a convergence radius of $\frac{1}{\mathrm{e}}$, we can rewrite Eq. (15) as

$$
Z_{e q}(\alpha)=1+\alpha^{\rho}+\mathscr{O}\left(\alpha^{2 \rho}\right) .
$$

Inserting this at the place of $Z$ in Eq. (10) (or any other ansatz for $Y$, in the limit of small $1-Z^{-1}$ ) yields

$$
Y=1+\beta \alpha^{\rho}+\mathscr{O}\left(\alpha^{2 \rho}\right) .
$$

Thus, in combination with a Newtonian rheology with viscosity $v$ for the undamaged material we obtain from (8) for the viscous deformation rate

$$
\dot{\varepsilon}_{v}=\frac{1}{v}\left(\sigma+\frac{\beta}{(E \lambda)^{\rho}} \sigma^{\rho+1}\right)
$$

which is the rheology of a regularized power-law fluid.

Integrating Eqs. (7) and (20), subject to initial conditions $\varepsilon_{d}=\varepsilon_{v}=0$, leads to typical curves as shown in Fig. 2. The creep curve exhibits a steeper slope at $t=0$ (primary creep), followed by a flattening phase (secondary creep), and a subsequent acceleration (tertiary creep). These three states of creep are typical for polycrystalline ice [17]. We conclude that our rheological model shows several typical properties of polycrystalline ice: A regularized power-law fluid behavior for the effective viscous deformation, and the typical creep phases. It seems thus that a combination of delayed-elastic and viscous deformations, subject to damage governed by delayed-elastic deformation, is a very promising ansatz for flow and damage modeling of polycrystalline ice.

\footnotetext{
2 The Lambert function $W:\left[-\frac{1}{\mathrm{e}}, \infty\right) \mapsto \mathbb{R}$ is the real solution of the equation $W(x) \exp [W(x)]=x$. For positive $x$, this solution is unique, whereas for $-\frac{1}{2} \leq x<0$ there are two solutions, from which by convention one uses that which is $\geq-1$ (so the function is continuous at $x=0$ ). See also Ref. [2].
} 


\subsection{Isotropic model: construction and calibration}

In the last section, the basic concepts of the fiber bundle damage model have been outlined, together with the rheological consequences, on a one-dimensional example. Now we need to find a simple way how this theory may be applied to a real three-dimensional flow problem. Simply applying it to one axis of deformation while ignoring the others would create an artificial, unwanted anisotropy. In consequence, we consider the most general isotropic deformation: A deformation consisting of isotropic shear and a superposed bulk deformation. Even though viscous deformations of glacier ice are nearly volume-preserving, there is experimental evidence that elastic deformations of polycrystalline ice have a non-negligible bulk component [17]. Both may be subject to damage, and they may influence each other. We therefore need to find a formulation which considers damage as a function of both bulk and shear deformation. Consequently, the two types of deformation are coupled via the damage effect $Z$, acting on both.

The importance of bulk and shear deformations is emphasized by the fact that the damage behavior of ice in tension and compression experiments is extremely different; the critical stresses observed in tension experiments are up to one order of magnitude smaller than those for compression [16].

We denote by $\varepsilon_{o c t}$ the delayed-elastic octahedral shear deformation, and by $\varepsilon_{t r}$ the trace of the delayedelastic deformation tensor. For the time evolution of both quantities, we postulate-keeping in mind the results of the previous section-

$$
\begin{aligned}
\dot{\varepsilon}_{o c t} & =\frac{1}{\eta}\left[Z\left(\varepsilon_{o c t}, \varepsilon_{t r}\right) \sigma_{o c t}^{D}-2 G \varepsilon_{o c t}\right], \\
\dot{\varepsilon}_{t r} & =\frac{1}{\eta}\left[Z\left(\varepsilon_{o c t}, \varepsilon_{t r}\right) \operatorname{tr}(\boldsymbol{\sigma})-K \varepsilon_{t r}\right],
\end{aligned}
$$

where $\boldsymbol{\sigma}$ is the Cauchy stress tensor, $\sigma_{o c t}^{D}$ the octahedral shear stress, and $G, K$ denote shear- and bulk moduli, respectively. Formulae (21), (22) are motivated by Eq. (3), and the moduli $G, K$ are suggested by the linear theory of elasticity. In order to provide more flexibility in adjustments with data, it is possible to mimic a nonlinear power-law creep by rewriting Eq. (21)

$$
\dot{\varepsilon}_{o c t}=\frac{1}{\eta}\left[Z\left(\varepsilon_{o c t}, \varepsilon_{t r}\right)\left(\frac{\sigma_{o c t}^{D}}{\sigma_{0}}\right)^{m-1} \sigma_{o c t}^{D}-2 G \varepsilon_{o c t}\right],
$$

where $\sigma_{0}$ is a constant that fixes the stress range which we consider (we will set it to $1 \mathrm{MPa}$ ). It will turn out that this yields slightly better fits of the experimental data; nevertheless, for the sake of simplicity we will keep formulation (21) throughout the rest of the article. General use of Eq. (23) would be possible though.

As for the damage effect $Z$, we propose

$$
Z\left(\varepsilon_{o c t}, \varepsilon_{t r}\right)=\exp \left[\left(\frac{\varepsilon_{o c t}}{\lambda}\right)^{\rho_{1}}+\left(\frac{\theta\left(\varepsilon_{t r}\right) \varepsilon_{t r}}{\mu}\right)^{\rho_{2}}\right]
$$

where $\theta$ denotes the Heaviside function. ${ }^{3}$ Thus, the damage effect is affected by shear deformation and by positive (i.e., tensional) bulk deformation. From the observation that the critical stresses in tension experiments are one order of magnitude smaller than those for compression situations, we conclude from Eq. (16) that the same holds for $\lambda$ and $\mu$.

\subsection{Non-monotonic deformation, non-physical healing}

The model as presented so far was based on the assumption that the damage variable $Z$ can be written as a function of the delayed-elastic deformation. However, sticking strictly to this assumption would yield nonphysical results for a stress history which allows the delayed-elastic deformation to decrease: Damage would decrease as well. Nevertheless, keeping in mind the fiber bundle motivation, it is clear how this shortcoming has to be cured. The relevant quantity for the damage variable is not the actual value of $\varepsilon_{d}$ at a given time, but the maximum value it has reached beforehand. The damage variable is a handy means of keeping track of this effect: It can simply be set to evolve as soon as $\varepsilon_{d}$ increases, and be held constant otherwise. Implementing

\footnotetext{
${ }^{3}$ For the shear deformation the Heaviside function is not needed, $\varepsilon_{o c t}$ being always nonnegative.
} 


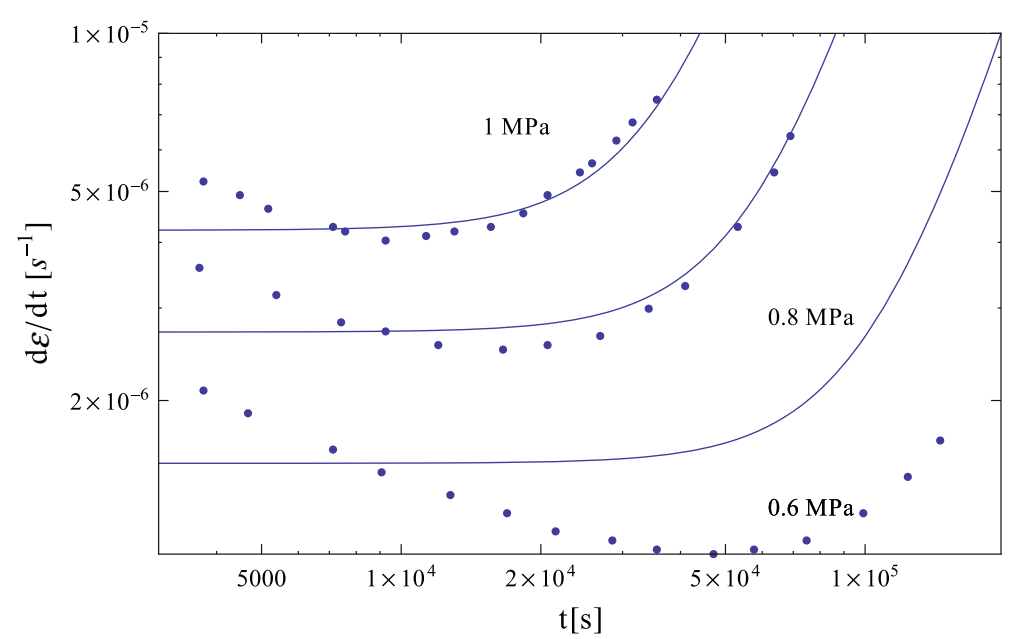

Fig. 3 Fit of the data by [6]. Dots: octahedral shear rates. Lines: fits with $\varepsilon(t)=\varepsilon_{d}+\varepsilon_{v}$ according to Eq. (23) and Eq. (24), with a power law with $m=2$. Parameters: $\eta=1.28 \times 10^{11} \mathrm{GPas}, \lambda=4.36 \times 10^{-4}, \beta=2.81 \times 10^{9}, v=2.37 \times 10^{5} \mathrm{GPas}$

such a non-monotonous model theoretically is somewhat tedious; the rate of the damage evolution has to be controlled such that it does not become negative. However, in a numerical implementation using time discretization, this can simply be reached by checking the evolution of damage in every time step. For this reason - and furthermore as for glaciological applications non-monotonous processes are far less interesting we will not pursue the topic of non-monotonous stress histories any further. It is to be understood that in any application of this damage model, the damage variable has numerically to be kept from decreasing.

Apart from the non-physical "healing" described above, it is likely that glacier ice actually shows a certain amount of damage reduction when subject to large compressive stresses. The description of this effect is an interesting theoretical task; as, however, to our knowledge hardly any experimental data is available on this effect, we have to refrain from constructing a healing model in this work. Particularly from the viewpoint of thermodynamics, healing is a very delicate subject. The thermodynamic framework which we will present in the second part of this work [9] will certainly help evaluating the thermodynamic correctness of any healing model developed in the future.

\section{Experimental data, calibration of the model}

Experiments exist mainly for uniaxial compression [6], and uniaxial tension [13]. It cannot be expected that these two inherently different stress and strain states are both described by the same one-dimensional model. However, the framework introduced above allows us to treat the two experiments more or less independently. During a compression experiment (with or without confining pressure), there will be no positive bulk deformation, that is, the evolution of the damage effect is given purely by shear deformation. Consequently, the two deformation components are not coupled, and an independent analysis of the shear component is feasible (a simultaneous analysis of shear and bulk deformation would be complicated by the fact that [6] does not provide any information on bulk deformation).

Reference [6] gives octahedral strain rates for different (constant) octahedral stresses and temperatures. We choose to fit the $-10 \operatorname{deg} \mathrm{C}$ data for stresses close to $1 \mathrm{MPa}$ with a numerical solution of $\dot{\varepsilon}=\dot{\varepsilon_{d}}+\dot{\varepsilon_{v}}$, using Eqs. (7) and (10) with $f(\sigma)=\frac{\sigma}{v}$, with $v=$ const. We set $\rho_{1}$ equal to 3 , and use an experimental value for the shear modulus, $G=3.52 \mathrm{GPa}$ [17]. We fit the experimental data both with the linear version of the theory, Eq. (21), and with the power-law variant Eq. (23), with an exponent $m=2$. The results are displayed in Figs. 3, and 4, respectively. It turns out that the power law yields a reasonable fit of the data, at least for stresses close to $1 \mathrm{MPa}$. And also the linear version yields results in the correct order of magnitude. In order to simplify the calculus, we will proceed in the following with the linear version; nevertheless, the power law could also be kept all the way through, and does not fundamentally change the exploitation of the entropy principle in part II [9].

On the other hand, in the tension situation considered by [13], the damage evolution is very likely to be dominated by the bulk deformation. Although these authors neither give any information on the amount of 


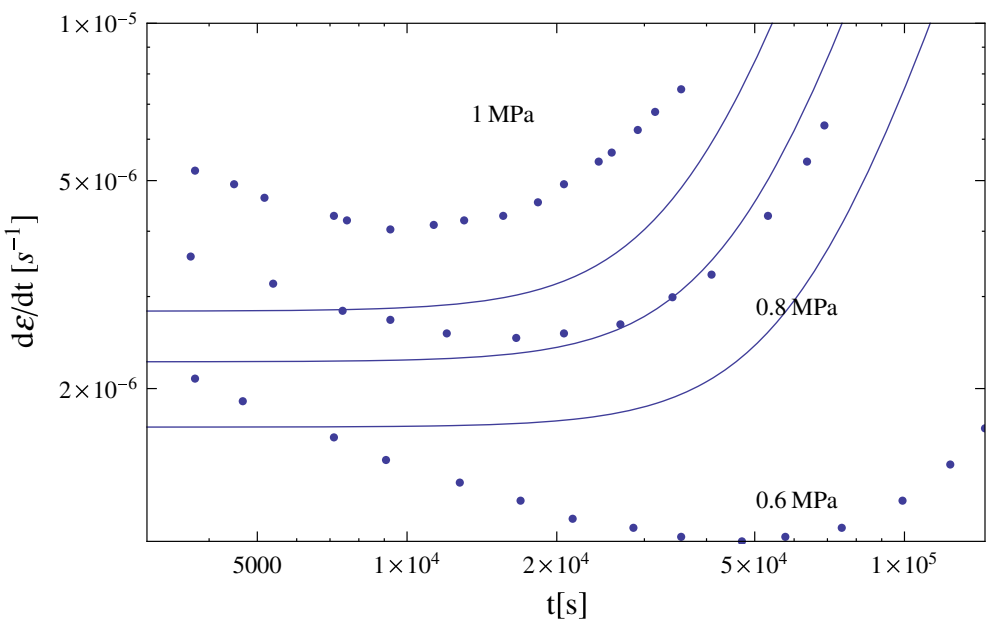

Fig. 4 Fit of the data by [6]. Dots: octahedral shear rates. Lines: fits with $\varepsilon(t)=\varepsilon_{d}+\varepsilon_{v}$ according to Eq. (21) and Eq. (24) (linear version). Parameters: $\eta=1.92 \times 10^{11}$ GPas, $\lambda=4.36 \times 10^{-4}, \beta=2.81 \times 10^{9}, v=2.37 \times 10^{5}$ GPas

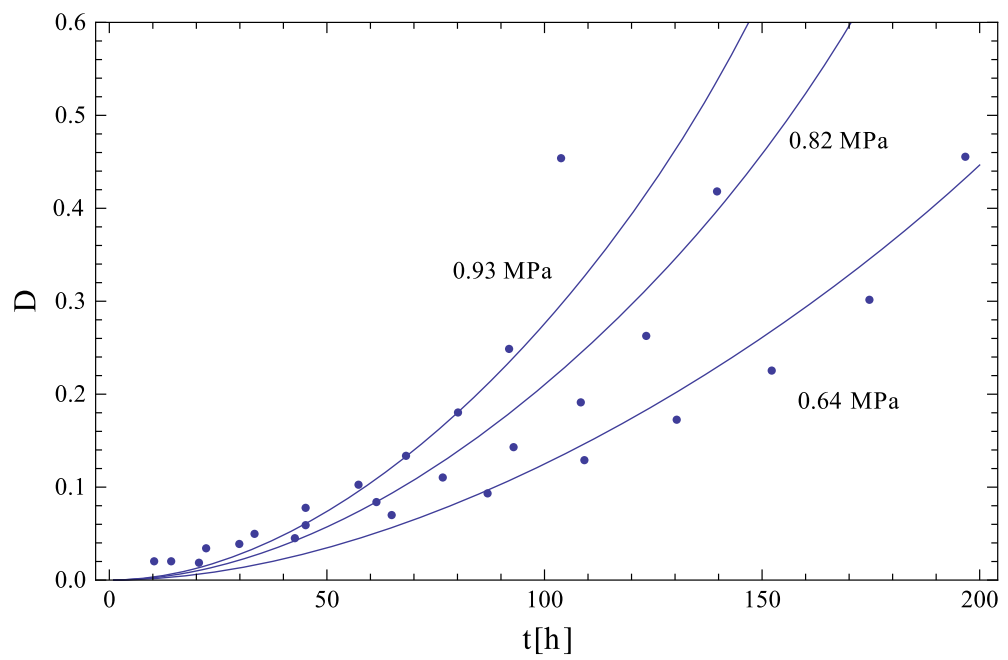

Fig. 5 Tension test at $\{0.93,0.82,0.64\} \mathrm{MPa}$. Fit of the data by [13]. Dots: data points. Lines: fits with $D=1-\frac{1}{Z\left(\varepsilon_{d}\right)}$. Parameters: $\mu=4.53 \times 10^{-5}$

bulk deformation actually observed, because of $\lambda \ll \mu$ we conclude that the damage evolution observed in their experiment must mainly be driven by delayed-elastic bulk deformation. Reference [13] gives data for a damage variable

$$
D=1-\frac{1}{Z}
$$

We fit it with $Z=Z\left(\varepsilon_{d}\right)$ as specified in Eq. (6), $\varepsilon_{d}$ being a numerical solution of Eq. (3). We set $\rho=2$, and use the bulk modulus $K=8.9 \mathrm{GPa}$ listed in [17]. We maintain the viscosity $\eta$ from the previous fit, and only fit the value for $\mu$. The results of the fits are displayed in Fig. 5. It turns out that the theory can produce a very reasonable fit of the data.

\section{General damage model}

In the last sections, we have motivated the construction of a fiber bundle damage model, and applied it to the simplest possible physical case, which is an isotropic model containing bulk and shear deformations. However, what has been presented so far does not provide a useful constitutive model yet. In order to obtain one, we have 
to properly define the degrees of freedom and the kinematics. This problem will be attacked in the following. Furthermore, we will at the same time generalize the model such that it may deal with induced anisotropy.

\subsection{Kinematics of viscous and delayed-elastic deformation}

In the one-dimensional example, elastic and viscous deformations have been coupled additively (Eq. (1)). If this coupling shall be extended to a continuum model, we have to find a suitable equivalent to this relation in terms of materially objective tensor quantities. We must find thus a suitable measure for elastic and viscous deformations which allows an additive coupling.

\subsubsection{The elastic degree of freedom}

In continuum mechanics, the motion of each particle of a given body is usually described by a time-dependent mapping of a reference (or Lagrangian) configuration $\mathscr{B}_{r}$ to a present (also called Eulerian) configuration $\mathscr{B}_{t}$. Each point of the physical body, considered as an abstract manifold, is identified with a point $X_{A} \in \mathbb{R}^{3}$ independently of time. The set of all points $X_{A}$ identified by the body points is called reference configuration $\mathscr{B}_{r}$; the reference coordinates $X_{A}$ "label" a certain material point of the body. The motion of a material point $X_{A} \in \mathscr{B}_{r}$ is described by a mapping

$$
\chi: \mathscr{B}_{r} \times \mathbb{R} \rightarrow \mathbb{R}^{3},\left(X_{A}, t\right) \mapsto x_{i}\left(X_{A}, t\right),\{A, i\} \subset\{1,2,3\} .
$$

The coordinates $x_{i}\left(X_{A}, t\right)$ describe the position of the material point $X_{A}$ at time $t$ in Euclidian space. The set of all present coordinates at a given time, $\chi(\mathscr{B}, t)$, is called present configuration at time $t$.

The description of elastic and viscous deformations proposed in this work consists in introducing a third configuration, which will be called the "viscous configuration." In order to distinguish between deformations which are purely elastic, and those due to viscous effects ${ }^{4}$, the coordinates of the present configuration are re-written as a viscous part $\mathbf{x}^{v}$ and a small delayed-elastic displacement $\mathbf{u}$,

$$
\mathbf{x}=\mathbf{x}^{v}+\mathbf{u}
$$

of which all three can be written as functions of the Lagrangian coordinates $X_{A}$. Both $\mathbf{x}$ and $\mathbf{x}^{v}$ are bijective functions of $X_{A}$, whereas $\mathbf{u}$ is not. This means, $\mathbf{x}$ and $\mathbf{x}^{v}$ may both serve as coordinates of the present configuration.

As measure of elastic deformations, the quantity

$$
F_{i j}^{d}=\frac{\partial x_{i}}{\partial x_{j}^{v}}=F_{i A} F_{A j}^{v-1}
$$

is used, where $F_{i A}=\frac{\partial x_{i}\left(X_{A}, t\right)}{\partial X_{A}}$ is the total deformation gradient, and $F_{i A}^{v}=\frac{\partial x_{i}^{v}}{\partial X_{A}}$ is the gradient of the viscous deformation. The quantity $F_{i j}^{d}$ can be interpreted as the displacement gradient of the entire deformation with respect to the viscous deformation; at the same time it is the transformation tensor that maps coordinates of vector quantities with respect to the $\mathbf{x}$-frame to those with respect to the $\mathbf{x}^{v}$-frame.

In order to find a useful constitutive variable for elastic deformation, the delayed-elastic Almansi tensor ${ }^{5}$ will be considered,

$$
A_{i j}^{d}=\frac{1}{2}\left(\delta_{i j}-B^{d-1}\right)
$$

where $B_{i j}^{d}=F_{i k}^{d} F_{j k}^{d}$ is the corresponding left Cauchy-Green deformation tensor. The Almansi tensor is an objective deformation measure [5]; it has both "legs" in the present configuration. For small elastic deformations (and the elastic deformations will always assumed to be small in the following), it equals to lowest order the linear strain tensor with respect to $\mathbf{x}^{v}$,

$$
\varepsilon_{i j}^{d}=\frac{1}{2}\left(\frac{\partial u_{i}}{\partial x_{j}^{v}}+\frac{\partial u_{j}}{\partial x_{i}^{v}}\right) .
$$

\footnotetext{
${ }^{4}$ Elastic means here that these deformations are completely recovered when the material is unloaded, whereas the viscous deformations will have non-vanishing residuals.

5 The superscript $d$ on $A^{d}$ will be omitted from now on, as no other Almansi tensor will be considered.
} 
Furthermore, to lowest order in $\varepsilon^{d}$, the volume change due to delayed-elastic deformation is

$$
\operatorname{det} \mathbf{F}^{d}=1+\operatorname{tr} \boldsymbol{\varepsilon}^{d} .
$$

Thus, for a volume-preserving material the trace of $\mathbf{A}$ is $\mathscr{O}\left(\varepsilon^{d^{2}}\right)$.

In consequence, for small deformations, the trace of the Almansi tensor can be identified with the bulk deformation, and the deviatoric part with volume-preserving deformations. It has to be stressed, however, that this approximation is not valid for large deformations. Nevertheless, in this work elastic deformations are generally considered as small; therefore, we will always consider tr $\mathbf{A}$ to measure bulk deformation.

\subsubsection{Coupling of delayed-elastic and viscous deformations}

Having set up now an additive splitting of viscous and elastic displacements, and a suitable measure of elastic deformation, it remains to find a suitable coupling of the two deformations. Keeping in mind that they are quadratic in the deformation gradients, it is clear that one cannot simply add the two Almansi tensors $\mathbf{A}^{d}$, $\mathbf{A}^{v}$ for the deformation components. It will turn out that the coupling has to be done not via the deformation tensors as such, but via the corresponding strain-rate tensors.

In the following, the time derivative of $\mathbf{A}$ will be calculated. This will both lead to an objective time derivative of $\mathbf{A}$, and to an additive coupling of the strain-rate tensors.

Starting from the definition Eq. (29), we can write the time derivative of the Almansi tensor as

$$
\dot{A}_{i j}=-\frac{1}{2} \frac{\mathrm{d}}{\mathrm{d} t}\left(F_{k i}^{d^{-1}} F_{k j}^{d^{-1}}\right) \text {. }
$$

Furthermore, it is straightforward to show that

$$
\begin{aligned}
\dot{F}_{i A} & =L_{i k} F_{k A}, \\
\dot{F}_{i A}^{v} & =\frac{\partial v_{i}^{v}}{\partial x_{k}^{v}} F_{k A}^{v}:=L_{i k}^{v} F_{k A}^{v},
\end{aligned}
$$

where $L_{i j}=\frac{\partial}{\partial x_{j}} \partial_{t} x_{i}\left(X_{A}, t\right)$ is the overall velocity gradient, $v_{i}^{v}=\partial_{t} x_{i}^{v}\left(X_{A}, t\right)$ is the velocity component due to viscous deformation as seen in the viscous deformation frame. These identities can be used to calculate

$$
\dot{F}_{i j}^{d}=L_{i k} F_{k j}^{d}-F_{i k}^{d} L_{k j}^{v} .
$$

Inserting this result into Eq. (32) yields the material time derivative of $\mathbf{A}$,

$$
\dot{\mathbf{A}}=\frac{1}{2}\left(\mathbf{L}+\mathbf{L}^{T}\right)-\mathbf{A} \mathbf{L}-\mathbf{L}^{T} \mathbf{A}-\frac{1}{2} \mathbf{F}^{d^{-T}}\left(\mathbf{L}^{v}+\mathbf{L}^{v T}\right) \mathbf{F}^{d^{-1}} .
$$

Thus, the total strain-rate tensor $\mathbf{D}=\frac{1}{2}\left(\mathbf{L}+\mathbf{L}^{T}\right)$ can be written as

$$
\mathbf{D}=\stackrel{\nabla}{\mathbf{A}}+\mathbf{D}^{v},
$$

where $\stackrel{\nabla}{\mathbf{A}}=\dot{\mathbf{A}}+\mathbf{A} \mathbf{L}+\mathbf{L}^{T} \mathbf{A}$ is the so-called Oldroyd-Derivative, an objective time derivative [4,5], and $\mathbf{D}^{v}=\frac{1}{2} \mathbf{F}^{d^{-T}}\left(\mathbf{L}^{v}+\mathbf{L}^{v T}\right) \mathbf{F}^{d^{-1}}$ is the viscous deformation gradient in the frame of the present configuration (the transformation tensor $\mathbf{F}^{d^{-1}}$ maps vector coordinates with respect to the $x^{v}$-frame onto those with respect to the $\mathbf{x}$-frame).

Equation (37) shows, how the two deformation contributions have to be coupled. One simply has to add up the two strain-rate tensors, $\stackrel{\nabla}{\mathbf{A}}$ and $\mathbf{D}^{v}$, to obtain $\mathbf{D}$.

A further consequence of this result is obtained if one considers its trace and deviatoric components. For a density-preserving fluid, the trace of the overall strain-rate tensor $\mathbf{D}$ vanishes. That is, in the presence of a strictly density-preserving viscous deformation, the necessary and sufficient condition for the elastic deformations to be density-preserving is

$$
\operatorname{tr} \stackrel{\nabla}{A}=0 .
$$

Unlike $\operatorname{tr} \mathbf{A}=0$, this relation is not an approximation, but is exact.

We have thus pointed out that using the delayed-elastic Almansi tensor $\mathbf{A}$ as an elastic degree of freedom allows an additive coupling similar to that of Eq. (1), with the difference that the coupling occurs within the strain rates, instead of the strains. 


\section{Constitutive model}

In the previous section, we have found the delayed-elastic Almansi tensor as a suitable objective measure of delayed elasticity, which allows an additive coupling of strain rates. In the following, we will point out how this finding can be used to construct a constitutive framework. This framework will allow the evolution of anisotropic damage in an originally isotropic material.

The damage variable will be represented by a rank-4 damage effect tensor $\mathbf{Z}$. The concept of a damage effect tensor is that it defines a linear mapping, which maps the Cauchy stress tensor on the corresponding effective stress (i.e., the tensor replacing the Cauchy stress in the constitutive relations of the damaged material).

\subsection{Free fields, constitutive relations}

Before starting to propose the constitutive framework, we must fix the set of free fields, and the constitutive variables which we are considering. There are different possible choices of these, and we have to be careful in selecting a reasonable setup.

The minimal choice of free fields is

$$
F=\{\mathbf{v}, \mathbf{A}, T,(p, \mathbf{Z})\} .
$$

which stand for velocity, delayed-elastic Almansi tensor, temperature and possibly pressure. The mass density does not appear in this set; this is due to the fact that it is a function of $\mathbf{A}$.

There are two free fields which one might add in certain cases (and therefore are written in parentheses in Eq. (39)). The first is the pressure $p$. This has to be considered for a density-preserving material. In this case, the velocity is divergence free; its 3 components are thus subject to a constraint. The constraint being non-holonomic, it cannot be used to eliminate one of the velocity components; therefore, a constraint force has to be taken into account. The pressure field supplies this constraint force field. As the density is coupled to $\mathbf{A}$, the number of degrees of freedom in $\mathbf{A}$ is in this case reduced to 5 .

The second additional variable is the rank-4 tensor field of the damage effect $\mathbf{Z}$. According to Sect. 2 , it is not a free field: It is rather coupled to the elastic deformation field $\mathbf{A}$. However, if non-monotonic stress histories are considered (i.e., the stress is also allowed to decrease), $\mathbf{Z}$ may still have to be treated as a free field: If it were coupled to $\mathbf{A}$, it would be likely that the damage effect decreases with decreasing stress. This is not physical: the material is not likely to "repair itself." Only in the presence of compressive stresses, a certain healing is suspected. Therefore, in this case the concerned components of $\mathbf{Z}$ have to evolve independently of A; either they are kept constant, or an evolution law is prescribed, which contains a healing parameterization. Consequently, the most general choice considered in this work is

$$
F=\{\mathbf{v}, \mathbf{A}, T, p, \mathbf{Z}\}
$$

In order to close the system of balance equations, constitutive relations have to be defined. These may depend on a certain set $S$ of free variables, called the free state space, which will be assumed to be

$$
S=\{\mathbf{D}, \mathbf{A}, T, \operatorname{grad} T\},
$$

where $\mathbf{D}$ is the overall deformation rate. If present as free variables, also $p$ and $\mathbf{Z}$ have to be included in $S$.

In the following, constitutive relations will be discussed for

- the Cauchy stress $\sigma$,

- the evolution law for $\mathbf{A}$,

$-\mathbf{Z}$ as a function of $\mathbf{A}$.

Apart from that, there have to be constitutive relations for the heat flux density and the internal energy; however, these are not the primary concern of this work; they are not likely to be different in the presence of damage from those in the virgin state. 


\subsection{Delayed-elastic deformation}

The basic concept of the evolution of delayed-elastic deformation has been discussed in a previous section. Now it is our task to construct an anisotropic generalization of this evolution law. In order to do so, the tensor analog of relations (21) and (22) will be considered,

$$
\begin{gathered}
\dot{\varepsilon}^{D}=\frac{1}{2 \eta_{D}}\left[Z_{D} \boldsymbol{\sigma}^{D}-2 G \boldsymbol{\varepsilon}^{D}\right], \\
\operatorname{tr} \dot{\varepsilon}=\frac{1}{\eta_{t r}}\left[Z_{t r} \operatorname{tr} \boldsymbol{\sigma}-K \operatorname{tr} \boldsymbol{\varepsilon}\right],
\end{gathered}
$$

where $\eta_{D}, \eta_{t r}$ are viscosities for elastic shear- and bulk deformations, and $G$ and $K$ are the corresponding elastic moduli. The superscript (.) ${ }^{D}$ identifies the deviatoric part of a rank-2 tensor, whereas a subscript (..) $D$ denotes a scalar quantity which is valid for the deviatoric part of the constitutive relation. Starting from these representations, a relation has to be constructed which is written in terms of the objective tensors $\mathbf{A}$ and $\stackrel{\nabla}{\mathbf{A}}$, involving a suitably defined rank-4 damage effect tensor $\mathbf{Z}=\mathbf{Z}(\mathbf{A})$.

There is no unique way how Eqs. (42), (43) have to be translated into evolution equations for A. Keeping in mind that for small deformations $\mathbf{A}$ agrees with $\boldsymbol{\varepsilon}$, it will be postulated that the separation of deviatoric and trace parts applies in the same way for $\mathbf{A}$,

$$
\stackrel{\nabla}{\mathbf{A}}=\frac{1}{2 \eta_{D}}\left[Z_{D} \boldsymbol{\sigma}^{D}-2 G \mathbf{A}^{D}\right]+\frac{1}{3 \eta_{t r}}\left[Z_{t r} \operatorname{tr} \boldsymbol{\sigma}-K \operatorname{tr} \mathbf{A}\right] \mathbb{1} .
$$

This relation is still not formulated in terms of an anisotropic damage effect tensor though. For the latter, the symmetry requirement of [8] has to be taken into account; thus, $\mathbf{Z}$ should be fully symmetric, and must not mix deviatoric and diagonal stresses. Therefore, it is reasonable to state

$$
\mathbf{Z}=\mathbf{Z}^{D}+\mathbb{1}_{t r} Z_{t r}
$$

where $\left(\mathbb{1}_{t r}\right)_{i j k l}=\delta_{i j} \delta_{k l}$ in Cartesian coordinates, and $Z_{t r} \in \mathbb{R}$. The deviatoric part $\mathbf{Z}^{D}$ maps deviatoric stresses on deviatoric stresses, i.e., $\operatorname{tr}\left(\mathbf{Z}^{D} \mathbf{B}\right)=0$ for any $\mathbf{B} \in \mathbb{R}^{3 \times 3}$ and $\mathbf{Z}^{D} \mathbb{1}=0_{3 \times 3}$. Both parts of $\mathbf{Z}$ may either depend on the components of $\mathbf{A}$, or may be governed by an evolution law.

Thus, the evolution law for $\mathbf{A}$ which generalizes Eq. (44) finally reads

$$
\stackrel{\nabla}{\mathbf{A}}=\frac{1}{2 \eta_{D}}\left[\mathbf{Z}^{D} \boldsymbol{\sigma}^{D}-2 G \mathbf{A}^{D}\right]+\frac{1}{3 \eta_{t r}}[\operatorname{tr} \mathbf{Z} \boldsymbol{\sigma}-K \operatorname{tr} \mathbf{A}] \mathbb{1} .
$$

If the two viscosities agree, $\eta_{D}=\eta_{t r}$, this can even be written as

$$
\stackrel{\nabla}{\mathbf{A}}=\frac{1}{2 \eta}[\mathbf{Z} \boldsymbol{\sigma}-\mathscr{C} \mathbf{A}],
$$

where $\mathscr{C}$ is a suitable isotropic "stiffness tensor" containing the elastic moduli.

\subsection{Damage}

As pointed out earlier, as a constitutive relation for the damage effect tensor, one can either specify a relation $\mathbf{Z}=\mathbf{Z}(\mathbf{A})$, or formulate an evolution law for $\mathbf{Z}$ as a dynamic degree of freedom. In the following the first task is addressed, followed by a brief discussion of the second.

The construction of an isotropic damage effect as a function of the delayed-elastic strain tensor has been discussed in Sect. 2. Starting from this result, we can write analogous relations for $Z_{t r}=Z_{D}$ in terms of $\mathbf{A}$ (compare with Eq. (24)),

$$
Z_{t r}=Z_{D}=\exp \left[\left(\frac{\operatorname{tr} \mathbf{A}}{\mu}\right)^{\rho_{1}}+\left(\frac{\sqrt{\operatorname{tr} \mathbf{A}^{\mathbf{D}} \mathbf{A}^{\mathbf{D}}}}{\lambda}\right)^{\rho_{2}}\right] .
$$


In order to find a suitable tensor relation for $\mathbf{Z}^{D}$, one has to look for a function mapping rank-2 deviators to deviatoric rank-4 tensors, which in the isotropic case (i.e., $\mathbf{A} \propto \boldsymbol{\sigma}^{D}$ ) reduces to

$$
\mathbf{Z}^{D} \boldsymbol{\sigma}^{D}=Z_{D} \boldsymbol{\sigma}^{D}
$$

with $Z$ as in Eq. (48).

A relation which fulfills this requirements is (at least for $\rho_{1}=2$ )

$$
\mathbf{Z}^{D}(\mathbf{A})=\exp \left[\left(\frac{\operatorname{tr} \mathbf{A}}{\mu}\right)^{\rho_{1}}\right]\left\{\operatorname{Exp}\left[\frac{\mathbf{A}^{D} \otimes \mathbf{A}^{D}}{\lambda^{2}}\right]-\frac{2}{3} \mathbb{1}_{\text {tr }}\right\} .
$$

Exp denotes the tensor exponential, and $\otimes$ the tensor product. The exponential is defined by the usual exponential series, where the $a$ th power of a rank-four tensor $\mathbf{W}$ has the coordinates

$$
\left(\mathbf{W}^{a}\right)_{i j k l}=W_{i j m_{1} n_{1}} W_{m_{1} n_{1} m_{2} n_{2}} \times \cdots \times W_{m_{a-1} n_{a-1} k l} .
$$

The additional $-\frac{2}{3} \mathbb{1}_{\text {tr }}$ guarantees that the 0 th order of the term in braces in Eq. (50) is deviatoric. Using explicitly the exponential series defining it, the tensor exponential in Eq. (50) can be rewritten as

$$
\begin{aligned}
\operatorname{Exp}\left[\frac{\mathbf{A}^{D} \otimes \mathbf{A}^{D}}{\lambda^{2}}\right]-\frac{2}{3} \mathbb{1}_{\text {tr }} & =\mathbb{1}-\frac{2}{3} \mathbb{1}_{\text {tr }}+\frac{\mathbf{A}^{D} \otimes \mathbf{A}^{D}}{\lambda^{2}}+\frac{1}{2} \frac{\operatorname{tr}\left[\left(\mathbf{A}^{D}\right)^{2}\right] \mathbf{A}^{D} \otimes \mathbf{A}^{D}}{\lambda^{4}}+\cdots \\
& =\mathbb{1}-\frac{2}{3} \mathbb{1}_{\mathrm{tr}}+\frac{\mathbf{A}^{D} \otimes \mathbf{A}^{D}}{\operatorname{tr}\left[\left(\mathbf{A}^{D}\right)^{2}\right]}\left\{\exp \left[\frac{\operatorname{tr}\left[\left(\mathbf{A}^{D}\right)^{2}\right]}{\lambda^{2}}\right]-1\right\} .
\end{aligned}
$$

With the use of this relation, it can be shown that for $\mathbf{A}^{D} \propto \boldsymbol{\sigma}^{D}$

$$
\mathbf{Z}^{D} \boldsymbol{\sigma}^{D}=Z_{D} \boldsymbol{\sigma}^{D}
$$

holds, with $Z_{D}$ as defined in Eq. (48). Thus, relation (50) has the correct isotropic behavior.

If maintaining the trace part according to Eq. (48), the tensor $\mathbf{Z}$ as a whole finally reads

$$
\begin{aligned}
\mathbf{Z}(\mathbf{A})= & \exp \left[\left(\frac{\operatorname{tr} \mathbf{A}}{\mu}\right)^{\rho_{1}}+\left(\frac{\sqrt{\operatorname{tr} \mathbf{A}^{\mathbf{D}} \mathbf{A}^{\mathbf{D}}}}{\lambda}\right)^{\rho_{2}}\right] \mathbb{1}_{t r} \\
& +\exp \left[\left(\frac{\operatorname{tr} \mathbf{A}}{\mu}\right)^{\rho_{1}}\right]\left\{\operatorname{Exp}\left[\frac{\mathbf{A}^{D} \otimes \mathbf{A}^{D}}{\lambda^{2}}\right]-\frac{2}{3} \mathbb{1}_{\mathrm{tr}}\right\} .
\end{aligned}
$$

\subsection{Enhanced viscous deformation}

The most important part of the damage model is again the constitutive formalism of the viscous deformation. In the following, basically a quasi-Newtonian fluid will be considered, the flow of which is enhanced by a damage-dependent tensor. That is, the viscous deformation rate is given by

$$
\mathbf{D}^{v}=f\left(I_{\sigma^{D}}\right) \mathbf{Y} \boldsymbol{\sigma}^{D},
$$

where $f$ is any scalar function; this could be, e.g., a constant or a polynomial (modified power law).

The difficulty consists again in a reasonable constitutive assumption for the rank-4 strain-rate enhancement tensor Y. Analogously to the one-dimensional case one could write either one of the relations

$$
\begin{aligned}
& \mathbf{Y}_{1}=\left[\mathbb{1}-\beta\left(\mathbb{1}-\mathbf{Z}^{-1}\right)\right]^{-1}, \\
& \mathbf{Y}_{2}=\mathbb{1}+\beta\left(\mathbb{1}-\mathbf{Z}^{-1}\right),
\end{aligned}
$$

with a constant factor $\beta$. The third proposal, Eq. (11), can only be used for $\mathbf{Z}$ sufficiently close to identity, as otherwise artanh cannot be written as a power series. In this case, however, all three proposals agree more or less anyway. 
In Eq. (56), no attention has been paid yet to deviatoric and trace parts of the damage effect tensor. However, as the two operate on complementary subspaces, it is clear that they can be inverted separately,

$$
\mathbf{Z}^{-1}=Z_{t r}^{-1} \mathbb{1}_{t r}+\left(\mathbf{Z}^{D}\right)^{-1}
$$

where $\left(\mathbf{Z}^{D}\right)^{-1}$ inverts only the restriction of $\mathbf{Z}^{D}$ on the subspace of deviators. Thus, in Eq. (56) $\mathbf{Z}$ can be replaced by $\mathbf{Z}^{D}$, as $\mathbf{Z}^{t r} \propto \mathbb{1}_{t r}$ maps $\boldsymbol{\sigma}^{D}$ to 0 . Thus, if we decide to use relation (57), together with a Newtonian rheology with viscosity $v$ for the un-damaged fluid, Eq. (55) may finally be re-written as

$$
\mathbf{D}^{v}=f\left(I_{\sigma^{D}}\right) \mathbf{Y} \boldsymbol{\sigma}^{D}=\frac{1}{2 v}\left[\mathbb{1}_{D}+\beta\left[\mathbb{1}_{\mathbb{D}}-\left(\mathbf{Z}^{D}\right)^{-1}\right]^{-1}\right] \boldsymbol{\sigma}^{D},
$$

which completes the constitutive framework for the viscous deformation.

\subsection{Coupling to the kinematic framework}

In Sect. 4.1, we have demonstrated how delayed-elastic and viscous deformations can reasonably be coupled such that both give a contribution to the overall velocity field.

As a result, it turned out that the Cauchy stresses have to be written in terms of the strain-rate tensor $\mathbf{D}$ and the Almansi strain tensor $\mathbf{A}$.

We start thus from the delayed-elastic-viscous coupling equation

$$
\mathbf{D}=\stackrel{\nabla}{\mathbf{A}}+\mathbf{D}^{v}
$$

inserting the constitutive relations Eq. (46) and Eq. (59) yields the result

$$
\mathbf{D}=\frac{1}{2 \eta_{D}}\left(\mathbf{Z}^{D} \boldsymbol{\sigma}^{D}-2 G \mathbf{A}^{D}\right)+\frac{1}{3 \eta_{t r}}[\operatorname{tr}(\mathbf{Z} \boldsymbol{\sigma})-K \operatorname{tr} \mathbf{A}] \mathbb{1}+\frac{1}{2 v} \mathbf{Y} \boldsymbol{\sigma}^{D} .
$$

Now the fact that the tensor $\mathbf{Z}$ (and therefore also $\mathbf{Y}$ ) does not mix trace- and deviatoric tensors has to be exploited. By using $\operatorname{tr}(\mathbf{Z} \sigma)=Z_{t r} \operatorname{tr} \sigma$, The last equation can be re-written as

$$
\mathbf{D}^{D}+\frac{1}{3} \operatorname{tr} \mathbf{D} \mathbb{1}=\left(\frac{1}{2 \eta_{D}} \mathbf{Z}^{D}+\frac{1}{2 v} \mathbf{Y}\right) \boldsymbol{\sigma}^{D}-\frac{G}{\eta_{D}} \mathbf{A}^{D}+\frac{1}{3 \eta_{t r}}\left(Z_{t r} \operatorname{tr} \boldsymbol{\sigma}-K \operatorname{tr} \mathbf{A}\right) \mathbb{1} .
$$

It is clear that $Z_{t r} \neq 0$. Even for the tensor $\left(\frac{1}{2 \eta_{D}} \mathbf{Z}^{D}+\frac{1}{2 \nu} \mathbf{Y}\right)$, it is reasonable to assume that it can be inverted (in the sense that its restriction to deviators can be inverted), as it is in most cases close to unity. Its inverse will be denoted by

$$
\mathbf{W}=\mathbf{W}(\mathbf{Z})=\left(\frac{1}{2 \eta_{D}} \mathbf{Z}^{D}+\frac{1}{2 v} \mathbf{Y}\right)^{-1}
$$

Therefore, the inverse relation of (62) reads

$$
\begin{gathered}
\operatorname{tr} \boldsymbol{\sigma}=\frac{\eta_{t r}}{Z_{t r}}\left(\operatorname{tr} \mathbf{D}+\frac{K}{\eta_{t r}} \operatorname{tr} \mathbf{A}\right), \\
\boldsymbol{\sigma}^{D}=\mathbf{W} \cdot\left(\mathbf{D}^{D}+\frac{G}{\eta_{D}} \mathbf{A}^{D}\right) .
\end{gathered}
$$

This relation gives the Cauchy stress in terms of $\mathbf{D}, \mathbf{A}$, and the damage effect tensor $\mathbf{Z}$ (which is either a free variable or a function of $\mathbf{A}$ ). It has been obtained as a result of the additive coupling of the strain-rate tensors. Together with the time evolution equation for $\mathbf{A}$, this turns the Stokes equation at a given time into an inhomogeneous spatial partial differential equation for the velocity field. 


\section{Conclusion}

In this article, we have discussed a new approach to model damage in polycrystalline (glacier) ice. Following an idea by [18], we have coupled the evolution of damage to the delayed-elastic component of the deformation, using a Weibull- distributed fiber bundle model for the construction of the damage function. In a one-dimensional example, we could demonstrate that this damage theory exhibits the phenomena of primary, secondary and tertiary creep, and a transition from stable to unstable damage evolution, depending on the stress level.

Furthermore, we have implemented this damage approach into an isotropic model of ice deformation, which has been calibrated on experimental ice creep and ice damage data.

Finally, we have pointed out how our damage approach can be included in a full constitutive framework. It turned out that the delayed-elastic Almansi tensor A is a suitable measure for the elastic deformation component; this choice not only fulfills the requirement of material frame independence, but also allows to couple elastic and viscous deformations via an additive coupling of the strain rates. Damage is represented by a rank-4 tensor, which is given as a function of the delayed-elastic Almansi tensor. This damage effect tensor has the structure required by Ref. [8] for reasons of thermodynamic consistency.

The resulting constitutive theory is able to deal with induced anisotropy of an originally isotropic fluid and to evolve and advect local elastic deformation and damage. Most of the considerations have been restricted to the case of monotonically increasing elastic deformations (and therefore damage) - the case which is more interesting to glaciological applications. In order to deal with decreasing elastic deformations, damage simply has to be held constant (which numerically can easily be implemented). What has not been considered so far is the effect of healing in the presence of strong compressive stresses. This effect should urgently be addressed by future work; however, this would also require further laboratory research.

As a next step, a thorough analysis of the thermodynamics of the proposed constitutive theory is required. This will be the subject of a subsequent publication [9].

Acknowledgments The work of A.K. has been funded by the Swiss National Science Foundation, Grant No. 20021-119781/1. We thank the anonymous referee for his review, which improved our paper.

\section{References}

1. Chaboche, J.L.: Thermodynamically founded CDM models for creep and other conditions. In: Altenbach, H., Skrzypek, J.J. (eds.) Creep and Damage in Materials and Structures, chap. 5., pp. 209-283. Springer, Wien, NY (1999)

2. Corless, R., Gonnet, G., Hare, D., Jeffrey, D., Knuth, D.: On the Lambert W-function. Adv. Comput. Math. 5(1), 329359 (1996)

3. Duddu, R., Waisman, H.: A temperature dependent creep damage model for polycrystalline ice. Mech. Mater. 46, 23$41(2012)$

4. Greve, R.: Kontinuumsmechanik. Springer, Berlin (2003)

5. Hutter, K., Jöhnk, K.: Continuum Methods of Physical Modeling: Continuum Mechanics, Dimensional Analysis, Turbulence. Springer, Berlin, Germany (2004)

6. Jacka, T.H.: The time and strain required for development of minimum strain rates in ice. Cold Regions Sci. Technol. 8(3), 261-268 (1984)

7. Kachanov, L.M.: Time of the rupture process under creep conditions (in Russian). Izv. Akad. Nauk. USSR, Otd. Tekh. Nauk., pp. 26-31 (1957)

8. Keller, A., Hutter, K.: On the thermodynamic consistency of the equivalence principle in continuum damage mechanics. J. Mech. Phys. Solids 59(5), 1115-1120 (2011). doi:10.1016/j.jmps.2011.01.015. http://www.sciencedirect.com/science/ article/B6TXB-523CDY6-2/2/bda2f1b70e114f23d673d5cbff6f7e1d

9. Keller, A., Hutter, K.: A viscoelastic damage model for polycrystalline ice, inspired by Weibull-distributed fiber bundle models. Part II: Thermodynamics of a rank-4 damage model. Continuum Mech. Thermodyn. doi:10.1007/s00161-014$0335-\mathrm{z}$

10. Krajcinovic, D.: Damage Mechanics. North-Holland, Amsterdam (1996)

11. Kun, F., Moreno, Y., Hidalgo, R., Herrmann, H.: Creep rupture has two universality classes. EPL (Europhys. Lett.) 63, 347 (2003)

12. Lemaitre, J., Lippmann, H.: A Course on Damage Mechanics, vol. 2. Springer, Berlin (1996)

13. Mahrenholtz, O., Wu, Z.: Determination of creep damage parameters for polycrystalline ice. Advances in Ice Technology (3rd Int. Conf. Ice Tech./Cambridge USA) pp. 181-192 (1992)

14. Nemat-Nasser, S., Hori, M.: Micromechanics: Overall Properties of Heterogeneous Materials, vol. 37. North-Holland, Amsterdam (1993)

15. Pralong, A., Hutter, K., Funk, M.: Anisotropic damage mechanics for viscoelastic ice. Contin. Mech. Thermodyn. 17(5), 387408 (2006). doi:10.1007/s00161-005-0002-5

16. Rist, M., Murrell, S.: Relationship between creep and fracture of ice. Mech. Creep-Brittle Mater. 2, 355-369 (1991) 
17. Schulson, E., Duval, P.: Creep and Fracture of Ice. Cambridge University Press, Cambridge (2009)

18. Sinha, N.K.: Crack-enhanced creep in polycrystalline material: strain-rate sensitive strength and deformation of ice. J. Mater. Sci. 23(12), 4415-4428 (1988)

19. Skrzypek, J.J., Ganczarski, A.: Modeling of Material Damage and Failure of Structures. Springer, Berlin (1999)

20. Szyszkowski, W., Glockner, P.: On a multiaxial constitutive law for ice. Mech. Mater. 5(1), 49-71 (1986)

21. Weiss, J., Gay, M.: Fracturing of ice under compression creep as revealed by a multifractal analysis. J. Geophys. Res. 103(B10), 24005-24 (1998)

22. Xiao, J., Jordaan, I.: Application of damage mechanics to ice failure in compression. Cold Regions Sci. Technol. 24(3), 305322 (1996) 